\title{
DISEÑO DE UN SISTEMA DE DISIPACIÓN PASIVA DE ENERGíA PARA UN EDIFICIO EN ALTURA DE GUAYAQUIL
}

\author{
DESIGN OF A PASSIVE ENERGY DISSIPATION SYSTEM \\ FOR A TALL BUILDING IN GUAYAQUIL
}

\section{GABRIEL PALAZZO', FRANCESC LÓPEZ-ALMANSA², VICTOR ROLDAN ${ }^{3}$, FRANCISCO CALDERÓN ${ }^{4}$}

\footnotetext{
1 Universidad Tecnológica Nacional, Argentina.gpalazzo@frm.utn.edu.ar

2 Universidad Politécnica de Cataluña, España. francesc.lopez-almansa@upc.edu

3 Universidad Tecnológica Nacional, Argentina. victor.roldan@frm.utn.edu.ar

4 Universidad Tecnológica Nacional, Argentina. francisco.calderon@frm.utn.edu.ar
}

RESUMEN

Se considera en este trabajo un edificio sismorresistente aporticado de hormigón armado, de 21 niveles, diseñado para Guayaquil, Ecuador. El objetivo del estudio es desarrollar un procedimiento de diseño de un sistema de disipación pasiva de energía (barras de pandeo restringido) para mejorar los parámetros de respuesta de la estructura original. Se diseñan los disipadores según el procedimiento que se explicita, y luego se realiza un análisis dinámico de historia en el tiempo para la estructura con / sin disipadores, bajo la excitación de 5 acelerogramas. Los resultados del modelo numérico muestran la eficiencia del sistema de disipación propuesto.

PALABRAS CLAVE: barras de pandeo restringido, diseño de sistemas de disipación de energía.
ABSTRACT In this work, we consider a seismic resistant building made of reinforced concrete, of 21 levels, designed for Guayaquil, Ecuador. The objective of the study is to develop a procedure for the design of a passive energy dissipation system (restricted bending bars) to improve the response parameters of the original structure. The dissipators are designed according to the procedure that is explained, and then a dynamic history analysis is performed for the structure with / without dissipators, under the excitation of 5 accelerograms. The results of the numerical model show the efficiency of the proposed dissipation system.

KEYWORDS: restricted buckling bars, design of energy dissipation systems. 
INTRODUCCIÓN

Este estudio toma como base un edificio de hormigón armado (HoAo) construido en Guayaquil (Ecuador), en una zona de elevada sismicidad. La geometría de un modelo computacional de esta construcción se presenta en la figura 1.

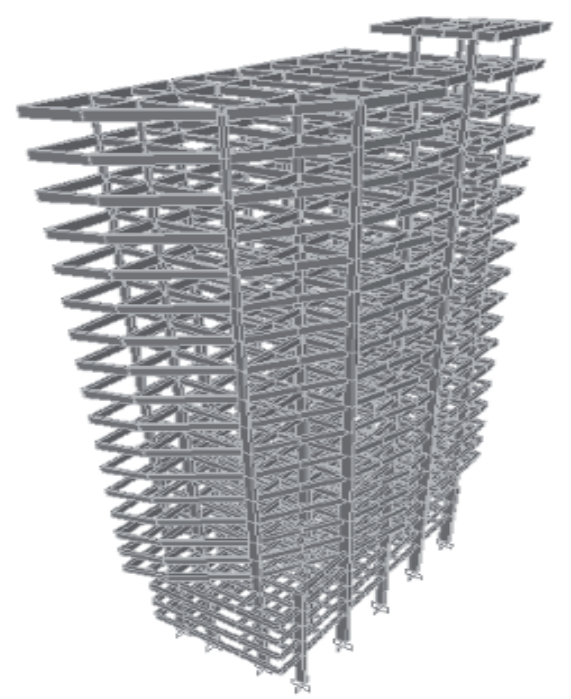

Figura 1. Geometría del modelo computacional del edificio en estudio.

Un estudio de ingeniería de Ecuador que realizó el diseño del edificio, consideró la opción de incorporar en la estructura principal un sistema de disipación pasiva de energía: las barras de pandeo restringido (BPR).

Numerosos estudios experimentales y numéricos han mostrado la eficiencia de los sistemas de disipación pasiva de energía para mejorar la respuesta de construcciones nuevas o existentes (Christopoulos y Filiatrault, 2006; Soong y Dargush, 1997; Housner et al, 1997). También se han reportado gran cantidad de edificaciones que han incorporado estos sistemas (Christopoulos y Filiatrault, 2006). Además, muchos países ya cuentan con prescripciones reglamentarias para la incorporación de dispositivos de disipación de energía (ASCE/SEI 7, 2010).

Las BPR, como la que se muestran en la Fig. 2 , son dispositivos de acero que ofrecen resistencia y disipación de energía a través de una plastificación bien distribuida en su volumen (en contraste con las riostras convencionales). Su configuración clásica consiste en un elemento de acero (transmite los esfuerzos axiales), un tubo de acero relleno de hormigón (para impedir el pandeo de la barra central), y una interfase entre la barra central y el hormigón para eliminar las transferencias de corte y la concentración de las deformaciones localizadas, permitiendo además la contracción lateral y la dilatación de la barra central al ser sometida a esfuerzos axiales.

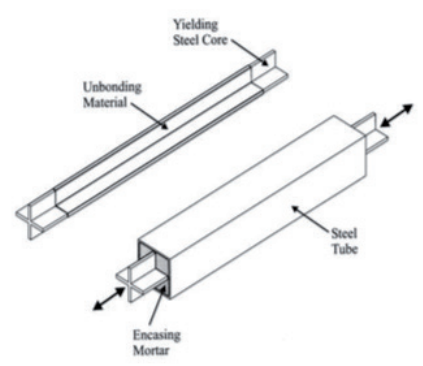

Figura 2. Esquema de una BPR.

Estos disipadores de energía pueden incorporarse como diagonales en vanos de pórticos tal como se presenta en la figura 3.

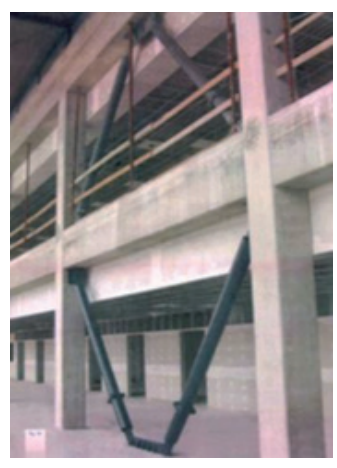

Figura 3. Sistema de BPR en estructura aporticada.

Las barras de pandeo restringido (Black et al 2002; López et al 2004; y Tremblay et al 2006), como un sistema de disipación pasiva histerético, también han sido ampliamente estudiadas; y se han implementado en varias construcciones.

Pero en el proyecto original de Ecuador no se logró un diseño de BPR que mejorara significativamente la respuesta de la estructura sin estos dispositivos. Por ello, el objetivo de este estudio es presentar una metodología de diseño de BPR con la que pueda alcanzarse el objetivo buscado.

La metodología de trabajo consiste en relevar parámetros de respuesta del modelo computacional de la estructura original, para luego (mediante un procedimiento iterativo) aplicar una metodología de diseño de BPR que permita mejorar la respuesta del edificio. El estudio numérico se realiza tomando como base el modelo computacional del edificio, desarrollado por el estudio de Ecuador; y considerando análisis dinámicos de historia en el tiempo, con acelerogramas escalados al espectro de Cuayaquil. En el diseño con BPR se consideró la limitación de ubicar disipadores de energía solo en los planos 
estructurales que habían dispuesto los ingenieros ecuatorianos.

En la sección 2 de este trabajo se presentan las características generales del edificio y del modelo computacional desarrollado por los ingenieros ecuatorianos; en la sección 3 se describen las BPR a considerar, y se desarrolla el procedimiento de diseño propuesto por los autores del trabajo; en la sección 4 se estudian y comparan los resultados numéricos de la estructura con / sin BPR; y en la sección 5 se presentan las conclusiones del trabajo.

\section{CARACTERIZACIÓN DEL EDIFICIO DE GUAYAQUIL} PARÁMETROS GEOMÉTRICOS GENERALES

El edificio cuya geometría general se mostró en la figura 1, tiene una estructura compuesta por pórticos sismorresistentes de $\mathrm{H}^{\circ} \mathrm{A}^{\circ}$ en las 2 direcciones principales. Posee 21 niveles más un cuerpo emergente, con una altura total de $78,95 \mathrm{~m}$. Cada nivel tiene, en general, un ancho de $16,10 \mathrm{~m}$ y un largo de $35,40 \mathrm{~m}$; con una superficie por planta de $570,00 \mathrm{~m}^{2}$, y una superficie cubierta total de $12000.00 \mathrm{~m} 2$. Las plantas típicas se muestran en la figura 4; mientras que en las figuras 5 y 6 se presentan vistas 2D en altura (en estas figuras la leyenda "plano" hace referencia a la denominación dada a cada plano estructural en la figura 4-a).

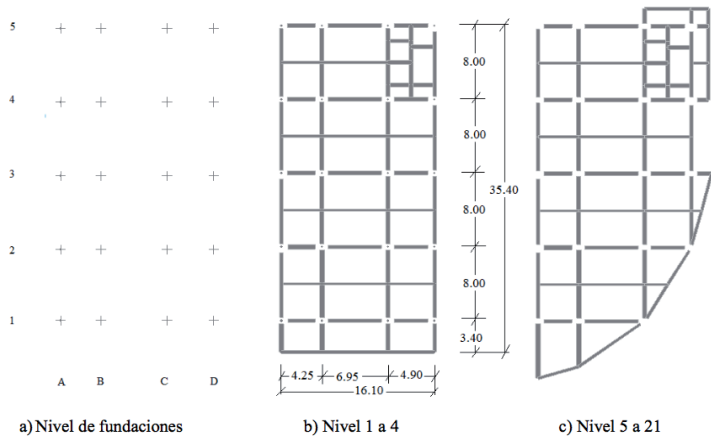

Figura 4. Plantas típicas del edificio.
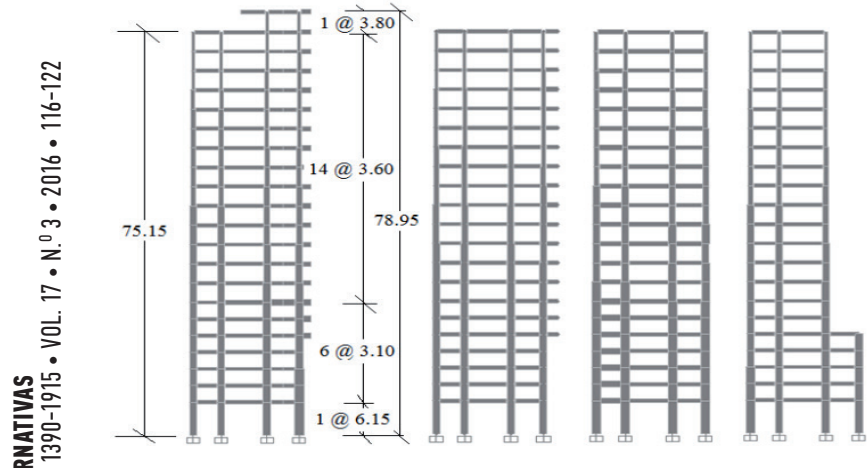

a) Plano 5 y 4

b) Plano 3

c) Plano 2

d) Plano 1

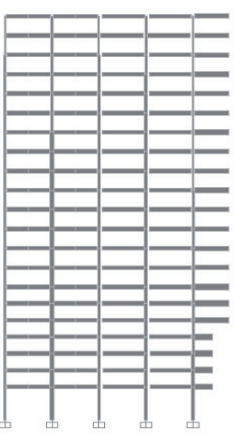

a) Plano A y B

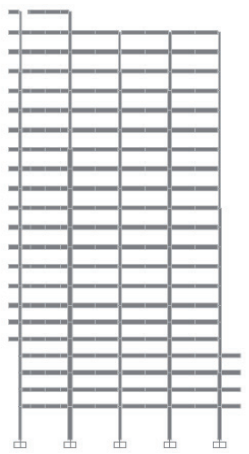

b) Plano C

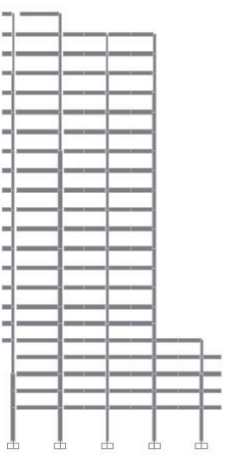

c) Plano D
Figura 6. Vistas en altura (dirección longitudinal).

Las dimensiones de las columnas de planta baja y del último nivel se presentan en la tabla 1 . Las vigas principales en planta baja tienen un ancho * alto (en [cm]) de $40 * 70$ y de $40 * 80$; mientras que las del último nivel de $30^{*} 70$ y de $30 * 80$.

TABLA 1. (A) DIMENSIONES DE COLUMNAS (ANCHO * ALTO) EN PRIMER NIVEL, EN [CM]

\begin{tabular}{rrrr} 
PLANO & A & B Y C & D \\
1 & $65 * 140$ & $50 * 110$ & $50 * 110$ \\
2 & $65 * 130$ & $65 * 130$ & $65 * 130$ \\
3 & $50 * 110$ & $50 * 110$ & $50 * 110$ \\
4 & $65 * 130$ & $65 * 130$ & $65 * 130$ \\
\hline 5 & $50 * 110$ & $50 * 110$ & $65 * 140$ \\
\hline
\end{tabular}

TABLA 1. (B) DIMENSIONES DE COLUMNAS (ANCHO * ALTO) EN ÚLTIMO NIVEL, EN [CM]

\begin{tabular}{rr} 
PLANO & A A D \\
\hline 1 & $30 * 45$ \\
2 & $50 * 60$ \\
\hline 3 & $30 * 45$ \\
\hline 4 & $50 * 60$ \\
\hline 5 & $30 * 45$ \\
\hline
\end{tabular}

MODELO COMPUTACIONAL DEL EDIFICIO

Para este trabajo se tomó como base el modelo estructural desarrollado por el estudio de ingeniería de Ecuador, cuya geometría general puede observarse en figura 1 , y en particular en las figuras. 4, 5 y 6 . Este modelo fue generado en un programa comercial de elementos finitos (SAP2000, 2010).

Para el HoAo de la estructura aporticada se consideró un material elástico lineal con un módulo de elasticidad longitudinal de 24821 $\mathrm{MPa}$, que corresponde a un hormigón con resistencia característica f'c de aprox. 28,00 MPa.

Para modelar vigas y columnas se dispusieron elementos frames; considerando para estas 
últimas empotramiento en sus bases. Las losas no fueron modeladas, pero se consideraron constraints para los nodos de un mismo nivel.

Se mantuvieron las cargas muertas y vivas del modelo Ecuador, que arrojan un peso total de la construcción de aprox. 149113,00 kN, que corresponde a una carga unitaria de aprox. $12,40 \mathrm{kN} / \mathrm{m}^{2}$.

Los períodos flexionales de los primeros dos modos en las direcciones principales se muestran en la tabla 2.

No se encontraron períodos torsionales o flexo-torsionales en los primeros doce modos calulados con el software de elementos finitos.

TABLA 2. PERÍODOS FLEXIONALES DEL MODELO, EN [S], PARA LOS PRIMEROS MODOS

\begin{tabular}{|c|c|c|}
\hline \multirow[t]{2}{*}{ MODO } & & DIRECCIÓN \\
\hline & Longitudinal & Transversal \\
\hline 1 & 3,86 & 3,17 \\
\hline 2 & 1,48 & 1,20 \\
\hline
\end{tabular}

EXCITACIÓN EN LA BASE

Cinco acelerogramas fueron considerados para el análisis dinámico de historia en el tiempo de la estrucura con / sin BPR. Las principales características de estos registros se mencionan en la tabla 3.

TABLA 3. CARACTERÍSTICAS DE LOS ACELEROGRAMAS CONSIDERADOS EN EL ESTUDIO NUMÉRICO

\begin{tabular}{rrrrr}
\hline DENOM. SISMO & FECHA & $\begin{array}{r}\text { MAGNITUD } \\
\text { DISTANCIA A } \\
\text { EPICENTRO } \\
\text { (KM) }\end{array}$ & $\begin{array}{r}\text { COMPONENTE PGA } \\
\text { (G) }\end{array}$ \\
\hline $\begin{array}{r}7036 \text { Huacho } \\
\text { - Perú }\end{array}$ & $16 / 10 / 1966$ & 8,1 & 150 & $-0,25$ \\
\hline 7038 Perú & $31 / 05 / 1970$ & 7,9 & 400 & 10,10 \\
\hline 7039 Perú & $31 / 05 / 1970$ & 7,9 & 400 & 20,09 \\
\hline 7050 Perú & 1974 & - & 250 & 10,18 \\
\hline 7051 Perú & 1974 & - & 250 & 20,16 \\
\hline
\end{tabular}

Los acelerogramas mencionados fueron escalados mediante un programa computacional (Seismomatch, 2013) al espectro de diseño de la norma ecuatoriana (NEC-11, 2011). El resultado de ese escalamiento se muestra en la figura 7. Con el software empleado, los espectros escalados para los períodos fundamentales en las direcciones transversal y longitudinal, caen por debajo del espectro de diseño. Pero considerando el objetivo principal de lograr un diseño con BPR más eficiente que para el caso de la estructura libre, no se realizó un nuevo escalamiento.

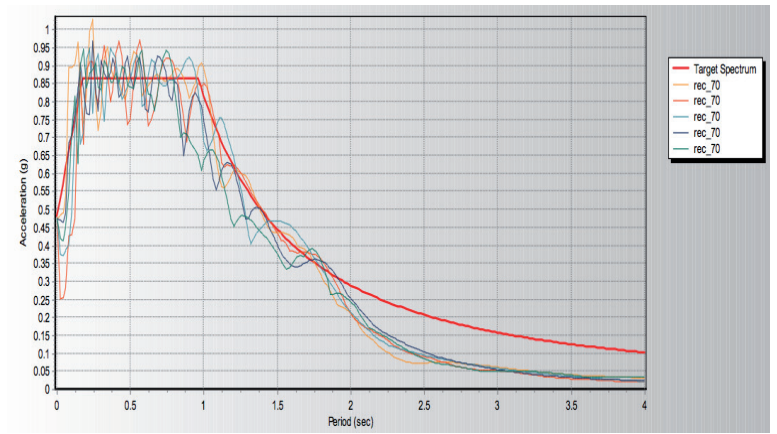

Figura 7. Espectro de diseño de la norma ecuatoriana vs. espectros de acelerogramas escalados.

PROCEDIMIENTO DE ANÁLISIS

Para el modelo de la estructura sin BPR se realizó con el programa (SAP2000, 2010) un análisis lineal de historia en el tiempo, considerando un amortiguamiento modal del 5\% para todos los modos.

\section{CARACTERIZACIÓN DE LAS BPR BPR SELECCIONADAS}

Las BPR adoptadas para el edificio Guayaquil están conformadas por una barra central de acero liso de sección circular, AL 220 (disponible en el mercado argentino), teflonadas y envueltas en una lámina de goma. El pandeo de la barra es evitado por un tubo de acero relleno con mortero. Uno de esos especímenes, desarrollado y ensayado por los autores (Palazzo et al, 2009), se muestra en la figura 8.

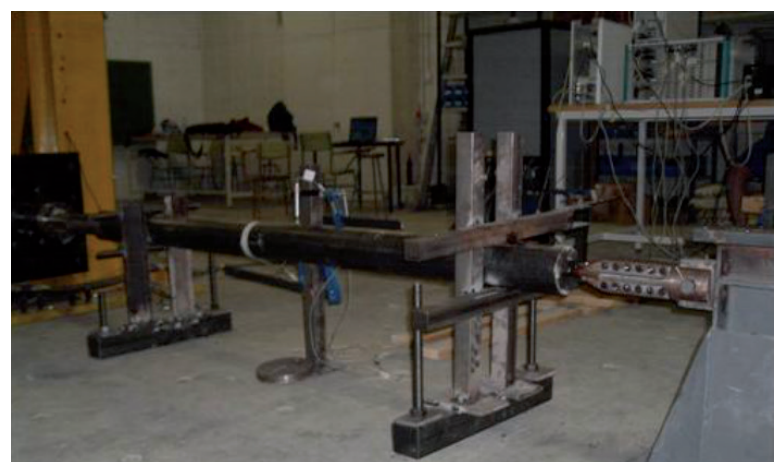

Figura 8. BPR propuesta.

DISPOSICIÓN DE LAS BPR EN EL EDIFICIO

Como ya se analizó, solo fue posible disponer BPR en los planos estructurales seleccionados inicialmente por los proyectistas de Ecuador. En la figura 9 se muestra la disposición de BPR en altura, tanto en la dirección transversal como en la dirección longitudinal. Se indica también en esa figura el diámetro de la barra central de la BPR para cada nivel, cuyo cálculo se explica en la sección 3.4. Se recuerda que la leyenda "plano" mencionada en figura 9 hace 
referencia a la denominación dada a cada plano estructural según la figura 4 - a.

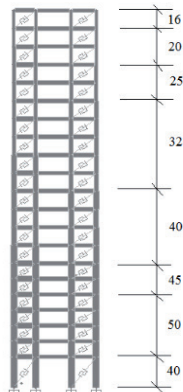

a) Plano 2 y 4

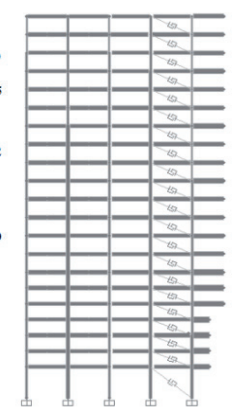

b) Plano A
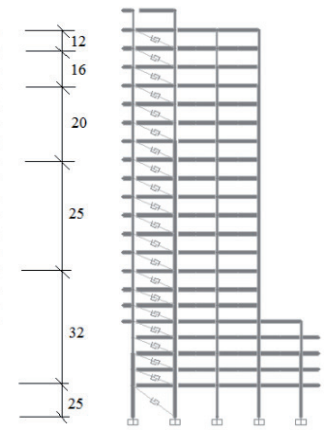

c) Plano D
Figura 9. Disposición de BPR en altura, y diámetros de las barras centrales consideradas (en [mm]).

MODELO NUMÉRICO DE LAS BPR

Para representar el comportamiento no lineal de la barra central de las BPR en el programa computacional (SAP2000, 2010), se emplearon elementos link según el modelo de plasticidad de Wen (expresado en fuerza - desplazamiento). Las propiedades introducidas en el programa para la dirección axial de cada disipador son: rigidez axial, fuerza en el inicio de la plastificación, relación de rigidez de post fluencia, y exponente de fluencia. Los primeros dos parámetros mencionados se determinan según se explica en la sección 3.4. La relación de rigidez de post fluencia se calculó según la tensión de fluencia del material, resistencia a tracción, deformación para el inicio de la fluencia, y deformación de rotura (según datos provistos por el proveedor del acero). En el caso del acero AL-220 se obtuvo una relación igual a 0,0033. Para el exponente de fluencia, que influye en la curvatura de la ley constitutiva al pasar del comportamiento elástico al plástico, se mantuvo el valor 2 predeterminado en el programa mencionado.

Dado que el sistema para evitar el pandeo de la barra central (tubo externo relleno de mortero) se encuentra desvinculado del comportamiento de la misma, no se considera este sistema en la simulación computacional.

\section{PROCEDIMIENTO DE DISEÑO DE LAS BPR}

El procedimiento de diseño implica determinar los dos parámetros principales que definen el modelo de plasticidad de Wen:

a. Rigidez axial de cada BPR (kBPR)

Para cada piso i, y para cada dirección principal, se calcula la rigidez horizontal que aporta el conjunto de BPR (KBPRs) en ese nivel y dirección, según la ec. (1).

$$
K_{\text {BPRs }}=C_{1} K_{P I s o}=K_{\text {Piso }}
$$

A partir de la matriz de rigidez de la estructura sin BPR, puede determinarse la rigidez horizontal de piso $K_{\text {Piso }}$. El coeficiente $C_{1}$ está en función del aumento de rigidez que proporcionan los disipadores, con un valor único para todo el edificio. Mediante un proceso iterativo basado en la comparación de resultados de la estructura con BPR, puede determinarse el valor óptimo de este parámetro. Para el edificio en estudio se llegó a $C_{1}=1,3$. Como estimación inicial pueden especificarse disipadores tal que la relación período fundamental de la estructura con riostra / período fundamental de la estructura libre sea cercana a 0,50 .

En función de la cantidad de BPR a colocar en cada nivel y plano estructural (No BPR), y del ángulo de inclinación $\alpha$ de la BPR respecto a la horizontal, se determina la rigidez que debe tener cada BPR en cada nivel $i, K_{B P R}$, según la ec. (2).

$$
K_{B P R}=\frac{K_{B P R s}}{N^{\mathrm{o}} B P R \cos ^{2} \alpha}
$$

Fuerza en el inicio de la plastificación de cada BPR (Fy)

Esta fuerza se calcula según la ec. (3).

$$
F_{y}=A_{y} F_{y}
$$

Donde:

$F_{y}$ : Tensión de fluencia del acero que conforma la barra central de la BPR.

$-A_{y}$ : Área de la zona de plastificación de la barra central, que se calcula con la ec. (8) siguiente.

Para calcular el área $A_{y}$ se considera la expresión de cálculo de la rigidez axial de una BPR con longitud $L$ y módulo de elasticidad $E$. Teniendo en cuenta que la barra posee una zona de plastificación (con área $A_{y}$ y longitud $L_{y}$ ), y otra zona de extremo para las conexiones (con área $A_{\text {extr }}$ y longitud $L_{\text {extr }}$ ), se puede expresar su rigidez axial según la ec. (4), suponiendo además que $A_{\text {extr }}=1,5 A_{y}$

$$
k_{B P R}=\frac{1}{\frac{L_{y}}{E A_{y}}+\frac{L_{\text {extr }}}{E A_{\text {extr }}}}=\frac{1}{\frac{L_{y}}{E A_{y}}+\frac{\left(L-L_{y}\right)}{E\left(1.5 A_{y}\right)}}
$$

La especificación de la longitud de plastificación $L_{y}$ se realiza según ley de Hooke, como se indica en la ec. (5).으

$$
L_{y}=\frac{E \Delta_{y}}{f_{y}} \leq\left(L-L_{e x t r}\right)
$$


Esta longitud es función del desplazamiento de plastificación $\Delta_{y}$, que se determina como un porcentaje del desplazamiento posible para la BPR, $\Delta_{P B R}$, según la ec. (6)

$$
\Delta_{y}=C_{2} \Delta_{B P R}
$$

Para el edificio en estudio se especificó $C_{2}=0,8$ y $\Delta_{P B R}$ según la deriva de piso $\Delta$ del nivel considerado, ec. (7).

$$
\Delta_{B P R}=\Delta \cos \alpha
$$

La deriva de piso $\Delta$ debe estimarse, por ejemplo en base a un análisis estático de la estructura original. Finalmente, y a partir de la ec. (4), puede calcularse el área de la zona de plastificación $A_{y}$ dada por la ec (8).

$$
A_{y}=\frac{\operatorname{KBPR}\left(L-0.5 L_{y}\right)}{1.5 E}
$$

Los diámetros de la zona de plastificación resultantes de la fase final del procedimiento iterativo anterior se indican en la figura 9.

ANÁLISIS DEL EDIFICIO CON LAS BPR

El modelo del edificio descripto en la sección 2, con las BPR consideradas en esta sección, fue sometido a los mismos acelerogramas escalados que se detallaron en la sección 2.3. Para la obtención de los valores de respuesta se realizó con el programa (SAP2000, 2010) un análisis no lineal de historia en el tiempo, donde la no linealidad solo estaba considerada en los elementos link relativos a las BPR.

ESTUDIO DE RESULTADOS

A modo de ejemplo, se muestra en la figura 10 la relación fuerza - desplazamiento que se obtienen en algunas BPR, para el edificio sometido al acelerograma 7036 (ver tabla 3). Para las otras BPR, y con la excitación de los otros acelerogramas, se obtienen curvas similares.

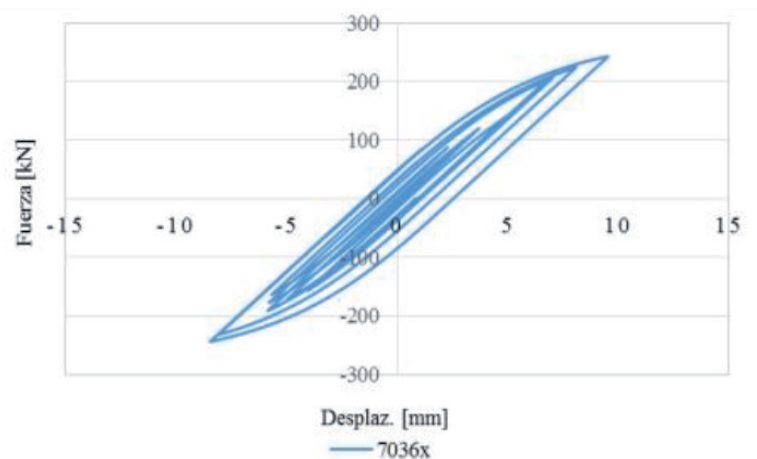

a) BPR en planta baja.

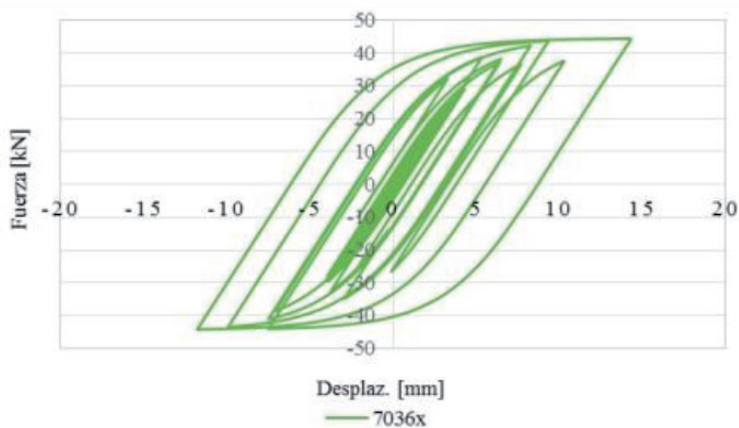

b) PBR en último nivel.

Figura 10. Relación fuerza - desplazamiento para BPR en edificio bajo la excitación del acelerograma 7036.

La cantidad de energía disipada en las BPR en función de la energía input, para las dos direcciones principales del edificio se indica en la tabla 4. Se observa que en la dirección transversal, donde se tienen dos BPR en cada nivel y plano estructural, la energía disipada duplica la energía disipada en la dirección longitudinal, donde se tiene una BPR en cada nivel y plano estructural.

TABLA 4. RELACIÓN ENERGÍA DISIPADA EN LAS BPRS / ENERGÍA INPUT, EN [\%]

\begin{tabular}{lrrrrr}
\hline DIRECCIÓN & \multicolumn{5}{c|}{ ACELEROGRAMA } \\
\cline { 2 - 6 } & 7036 & 7038 & 7039 & 7050 & 7051 \\
\hline Transv. & 29,6 & 27,9 & 30,4 & 25,6 & 26,7 \\
\hline Long & 12,6 & 12,0 & 11,9 & 9,3 & 11,2 \\
\hline
\end{tabular}

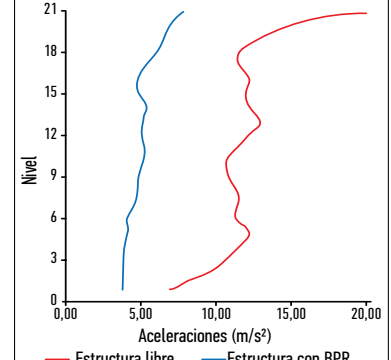

— Estructura libre —Estructura con BPR a) Dirección transversal

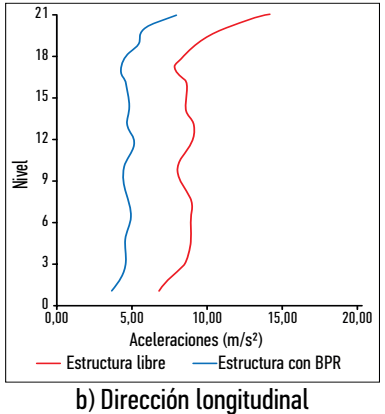

b) Dirección longitudinal
Figura 11. Aceleraciones máximas promedio, estructura con/sin BPR, bajo la excitación de 5 acelerogramas.

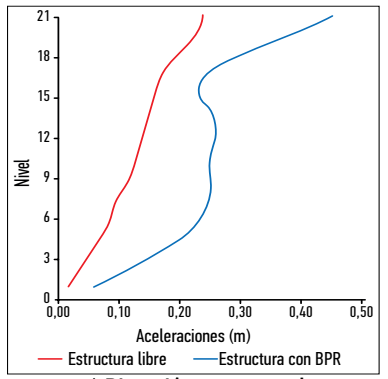

a) Dirección transversal

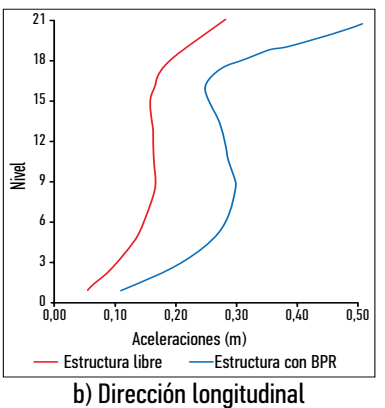

b) Dirección longitudinal
Figura 12. Desplazamientos máximos promedio, estructura con/sin BPR, bajo la excitación de 5 acelerogramas. 
La comparación entre los valores máximos promedio de aceleraciones y desplazamientos para la estructura con/sin BPR, excitada según los cinco acelerogramas detallados en tabla 3 , se presenta en las figuras 11 y 12 .

Para ambos parámetros de respuesta considerados, la reducción que se logra cuando se incorporan BPR en la estructura, es significativa. A pesar que en la dirección transversal la cantidad de energía disipada en las BPR duplica la energía disipada en la dirección longitudinal, no se observa una reducción de ese orden en los parámetros de respuesta evaluados.

CONCLUSIONES

Se ha considerado en este estudio un edificio aporticado de $\mathrm{H}^{\circ} \mathrm{A}^{\circ}$ de 21 niveles, sismorresistente, construido en Guayaquil, Ecuador. Mediante un procedimiento racional de diseño, iterativo, se logró alcanzar el objetivo de reducir en forma significativa los parámetros de diseño para la estructura con un sistema de disipación pasiva de energía.

REFERENCIAS BIBLIOGRÁFICAS

ASCE/SEI 7 (2010): Minimun design loads for buildings and other structures. American Society of Civil Engineers.

Black C, Makris N, Aiken L (2002): Component testing, stability analysis characterization of bucklingrestrained unbonded braces. Pacific Earthquake
Engineering Research Center, report PEER 2002/08. Christopoulos C and Filiatrault A (2006): Principles of passive supplemental damping and seismic isolation. IUSS Press.

Housner GW, Bergman LA, Caughey TK, Chassiakos AG, Claus RO, Masri SF (1997): Structural control: Past, present, and future. J Eng Mech ASCE; 123:897_971.

López WA, Gwie DS, Lauck TW, Saunders M. (2004): Structural design and experimental verification of a buckling-restrained braced frame system. Eng J; 41.

NEC-11 (2011): Norma ecuatoriana de la construcción. Comité ejecutivo de la norma ecuatoriana de la construcción.

Palazzo G, López Almansa F, Cahís J, y Crisafulli F (2009): A low-tech dissipative buckling restrained brace. Design, analysis, production and testing. Engineering Structures, Vol. 31, No 9, 2152-2161. SAP2000, v.14.2.4 (2010): Structural analysis program. Computers and Structures, Inc.

Seismomatch (2013): It is an application capable of adjusting earthquake accelerograms to match a specific target response spectrum. Seismosoft Ltd.

Soong T, Dargush G (1997): Passive energy dissipation systems in structural engineering. John Wiley. Tremblay R, Bolduc P, Neville R, De Vall R. (2006): Seismic testing and performance of buckling- restrained bracing systems. Canad J Civil Eng; 33:183_98. 